\title{
Singular robustly chain transitive sets are singular volume partial hyperbolic
}

\author{
Adriana da Luz
}

October 24, 2018

\begin{abstract}
For diffeomorphisms or for non-singular flows, there are many results relating properties persistent under $\mathcal{C}^{1}$ perturbations and a global structures for the dynamics ( such as hyperbolicity, partial hyperbolicity, dominated splitting). However, a difículty appears when a robust property of a ow holds on a set containing recurrent orbits accumulating a singular point.

In $\mathrm{BdL}$ with Christan Bonatti we propose a a general procedure for adapting the usual hyperbolic structures to the singularities.

Using this tool, we recover the results in BDP for flows, showing that robustly chain transitive sets have a weak form of hyperbolicity. allowing us to conclude as well the kind of hyperbolicity carried by the examples in BLY] (a robust chaintransitive singular attractor with periodic orbits of different indexes).

Along with the results in $\mathrm{BdL}$, this shows that the way we propose to interpret the effect of singularities, has the potential to adapt to other settings in which there is coexistence of singularities and regular orbits with the goal of reobtaining the results that we already know for diffeomorphisms.
\end{abstract}

Mathematics Subject Classification: AMS 37D30, 37D50

Keywords Multisingular singular partial hyperbolicity, dominated splitting, linear Poincaré flow, flows with singularities.

\section{Introduction}

\subsection{General setting and historical presentation}

While studying nature, the parameters of the systems in question will be determined by measurements that, for the fact of being made by humans, will introduce some error. It is natural to ask how resistant our conclusions are to this inevitable constrain. A robust properties is a property that is impossible to break by small perturbations of a system; in other words, a dynamical property is robust if its holds on a (non-empty) $\mathcal{C}^{1}$ open set of diffeomorphisms or flows. 
If the goal is then to study systems that present robust properties, we need to ask which robust properties are we interested in.

In this regard we might not be interested in all the orbits in our manifold, but rather in a subset, that can be shown to contain the relevant dynamical information: that is the chain recurrence classes. For this we consider pseudo orbits, that is a generalization of an orbit but at every iterate we allow an $\epsilon$ mistake or jump, then follow this new orbit (a precise definition is presented in the preliminaries section). In this sense, the chain recurrence set is the set of recurrent pseudo orbits, and the chain recurrence classes are compact invariant sets of points that can be connected by pseudo orbits for any $\epsilon$.

It is shown by Conley in $\mathrm{Co}$ that this chain classes play the role of fundamental pieces of the dynamics, and the rest of the orbits, simply go from one of this pieces to the other.

Recall that a maximal invariant set is the intersection of the iterates of an open set. And a chain recurrence class $C$ is said to be robustly transitive if there is a neighborhood $U \subset M$ and a neighborhood of $f, \mathcal{U}$ such that the maximal invariant set in $U$ is a unique chain class $C$ and has a dense orbit for any $g \in \mathcal{U}$. The definition of robustly chain transitive set is a generalization of this notion since it is equivalent to ask only that there is a neighborhood $U \subset M$ and a neighborhood of $f, \mathcal{U}$ such that the maximal invariant set in $U$ is a unique chain class $C$ for any $g \in \mathcal{U}$.

Reasoning as in the beginning of this introduction, it is also natural to ask ourselves: What is the situation when there are no robust properties?

In a series of papers first by Mañé [Ma, for surfaces and then [DPU] for 3 manifolds and culminating with $\mathrm{BDP}$ for the more general result, a dichotomy is presented between some hyperbolic like property and the appearance by perturbation of infinitely many sinks and sources. This shows that a Chain recurrence class might split under perturbation into infinitely many classes and there is no topological dynamical property that was preserved in this process.

The weak hyperbolic structure that forbids the appearance by perturbation of infinitely many sinks and sources was introduced by Mañé and Liao and is called dominated splitting:

Definition 1. Let $f: M \rightarrow M$ be a diffeomorphism of a Riemannian manifold $M$ and $K \subset M$ a compact invariant set of $f$, that is $f(K)=K$. A splitting $T_{x} M=E(x) \oplus F(x)$, for $x \in K$, is called dominated if

- $\operatorname{dim}(E(x))$ is independent of $x \in K$ and this dimension is called the $s$ index of the splitting;

- it is $D f$-invariant: $E(f(x))=D f(E(x))$ and $F(f(x))=D f(F(x))$ for every $x \in K$;

- there is $n>0$ so that for every $x$ in $K$ and every unit vectors $u \in E(x)$ and $v \in F(x)$ one has

$$
\left\|D f^{n}(u)\right\| \leq \frac{1}{2}\left\|D f^{n}(v)\right\| .
$$


One denotes $\left.T M\right|_{K}=E \oplus_{<} F$ the dominated splitting.

The results in $\mathrm{BDP}$ is as follows

Theorem 1. (theorem 1 in $[B D P])$ Let $P$ be a hyperbolic saddle of a diffeomorphism $f$ defined on a compact manifold $M$. Then

- either the homoclinic class $H(P, f)$ of $P$ admits a dominated splitting,

- or given any neighborhood $U$ of $H(P, f)$ and any $k \in \mathbb{N}$ there is $g$ arbitrarily $\mathcal{C}^{1}$-close to $f$ having $k$ sources or sinks, whose orbits are included in $U$.

Theorem 2. (theorem 2 in $[B D P]$ ) Every $C^{1}$-robustly transitive set (or diffeomorphism) admits a dominated splitting.

The conclusion also holds for an open and dense subset of vector fields and robustly chain transitive sets. This shows us that asking that the chain recurrence classes are preserved by perturbation induces a constraint in the dynamics of the vector field, that is, there must be a hyperbolic like property in the tangent space.

A generalization of this result implying the version for flows was given by BGV. Also in $\overline{B D P}$ it is shown that a robustly chain transitive sets must have a weak hyperbolic structure, that is :

Theorem 3. (theorem $4[B D P]$ ) Let $\Lambda_{f}(U)$ be a $C^{1}$-robustly transitive set and $E^{1} \oplus \cdots \oplus E^{k}$, be its finest dominated splitting. Then there exists $n \in \mathbb{N}$ such that $D f^{n}$ contracts uniformly the volume in $E^{1}$ and expands uniformly the volume in $E^{k}$.

A set $K$ is volume partial hyperbolic if there is a dominated splitting $E^{c s} \oplus$ $E^{c} \oplus E^{c u}$ so that the volume in $E^{c s}$ is uniformly contracted and the volume in $E^{c u}$ is uniformly expanded.

The aim of this paper is to generalize this results to flows with singularities. But the fact that in fifteen years this has not been done, gives the idea that there might be additional difficulties to deal with in this scenario. Firstly, a direct generalization of theorem 1 is not possible even for non singular flows, there are transitive sets that do not have a dominated splitting for the tangent space (for instance take the suspension of the example in [BV]) many hyperbolic structures for flows are not expressed in terms of the differential of the flow, but on its transverse structure (called the linear Poincaré flow). For non singular flows it has been shone that the results in 1 and 2 can be generalized (in $[\mathrm{V}$, [BGV] and [D]).

However the linear Poincaré flow is only defined far from the singularities, and therefore it cannot be used directly for understanding our problem. And there are in fact open sets of vector fields having robustly chain transitive singular chain recurrence classes. 
- There are many examples of singular robustly chain transitive singular chain recurrence classes, with the extra property of having all periodic orbits robustly hyperbolic that we called star flows (For instance the Lorenz attractor or very many others). In this cases much is known of their hyperbolic structures. see for instance [MPP, GLW], GSW] and [BdL].

- In BLY The authors present an example of a vector field with a chain transitive attractor in dimension 4 . This attractor has periodic orbits of different stable index and singularities. This example is not a star flow.

- The example just mentioned can be multiplied by a strong expansion for it not to be an attractor any more (now the example would be in a manifold of dimension 5).

For the last two items the kind of weak hiperbolicity they carry or not was yet unknown. However it is also evident that there are not that many examples of nontrivial singular chain recurrence clases out of the star flow setting.

In GLW], the authors define the notion of extended linear Poincaré flow defined on some sort of blow-up of the singularities. The extension of the chain recurrence class $\Lambda$ in $U$ with the blow-up, will be noted $\widetilde{\Lambda}$.

The definition of this blow up of the singularity relies on information of the perturbations of the vector field, so that this set varies upper semi continuously with the vector field in the $\mathcal{C}^{1}$ topology

But in $\mathrm{BdL}$ we propose a bigger set that (as opposed to the first case) does not depend on knowing any information from the neighbor vector fields. This set will be called extended maximal invariant and noted $B(X, U)$. We belive a dominated splitting should be a property that one can check without information of the surrounding vector fields.

Our notion of singular volume hyperbolicity will be expressed as the volume hyperbolicity of a well chosen reparametrization of this extended linear Poincaré flow over $B(X, U)$. But it is enough to do it over $\widetilde{\Lambda}$, since in $[\mathrm{BdL}]$ it is shown that they both carry the same hyperbolic properties.

\subsection{The singular volume partial hyperbolicity}

In this section we take a closer look at weaker forms of hyperbolicity and their relation with the persistence of the dynamical properties.

Following the proofs in $\mathrm{BDP}$, we show the following

Proposition 4. Let $\mathcal{U} \subset \mathcal{X}^{1}(M)$ be a $C^{1}$-open set such that, for every $X \in$ $\mathcal{U}$ there is an open set $U$ of $M$ such that the maximal invariant set in $U$ is an isolated chain recurrence class $C$. Then $\widetilde{\Lambda}$ has a uniform finest dominated splitting for the linear Poincaré flow:

$$
\mathcal{N}_{L}=\mathcal{N}_{L}^{1} \oplus \cdots \oplus \mathcal{N}_{L}^{n} .
$$

Each of this periodic orbits is volume partial hyperbolic for the tangent space. 
We divide the singular set $\operatorname{Sing}(X) \cap U$ in subsets sets:

- the set $S_{E c}$ of singular points whose escaping stable space has dimension smaller than $\mathcal{N}_{L}^{1}$,

- the set $S_{E}$ of singular points whose escaping stable space has dimension bigger or equal than $\mathcal{N}_{L}^{1}$,

- the set $S_{F c}$ of singular points whose escaping unstable space has dimension smaller than $\mathcal{N}_{L}^{n}$,

- the set $S_{F}$ of singular points whose escaping unstable space has dimension bigger or equal than $\mathcal{N}_{L}^{n}$.

We want:

- to reparametrize the cocycle $\psi_{\mathcal{N}}^{t}$ in restriction to $\mathcal{N}_{L}^{1}$ by the expansion in the direction $L$ if and only if the line $L$ is based at a point close to $S_{E c}$;

- to reparametrize the cocycle $\psi_{\mathcal{N}}^{t}$ in restriction to $\mathcal{N}_{L}^{n}$ by the expansion in the direction $L$ if and only if the line $L$ is based at a point close to $S_{F c}$.

For this we use again the cocycle center-stable cocycle $\left\{h_{E c}^{t}\right\}_{t \in \mathbb{R}}$,so that:

- $h_{E c}^{t}(L)$ and $\frac{1}{h_{E c}^{t}}(L)$ are uniformly bounded (independently of $t$ ), if $L$ is based on a point $x$ so that $x$ and $\phi^{t}(x)$ are out of a small neighborhood of $S_{E c}$, where $\phi^{t}$ denotes the flow of $X$;

- $h_{E c}^{t}(L)$ is in a bounded ratio with the expansion of $\phi^{t}$ in the direction $L$, if $L$ is based at a point $x$ so that $x$ and $\phi^{t}(x)$ are out of a small neighborhood of $S_{E}$.

- $h_{E c}^{t}$ depends continuously on $X$.

Analogously we get the notion of center-unstable cocycles $\left\{h_{F c}^{t}\right\}$ by exchanging the roles of $S_{E c}$ and $S_{F c}$ in the properties above.

Now similarly to the multisingular hyperbolicity case, we define the singular volume partial hyperbolicity.

Definition 2. Let $X$ be a $C^{1}$-vector field on a closed manifold $M$. Let $U$ be a compact set. We say that $X$ is singular volume partial hyperbolic in $U$ if:

- the extended linear Poincaré flow admits a finest dominated splitting $\mathcal{N}_{L}=$ $\mathcal{N}_{L}^{1} \oplus \cdots \oplus \mathcal{N}_{L}^{n}$. over the pre extended maximal invariant set $\widetilde{\Lambda}$.

- the set of singular points in $U$ is the union of

$$
S_{E c} \cup S_{F c} \cup S_{E} \cup S_{F},
$$

defined above.

- the reparametrized linear Poincaré flow $h_{E c}^{t} \psi_{\mathcal{N}}^{t}$ contracts volume on $\mathcal{N}_{L}^{1}$, where $h_{E c}^{t}$ is a center-stable cocycle,

- the reparametrized linear Poincaré flow $h_{F c}^{t} \psi_{\mathcal{N}}^{t}$ contracts volume on $\mathcal{N}_{L}^{n}$, where $h_{F c}^{t}$ is a center-unstable cocycle, 


\subsection{Robustly chain transitive singular sets}

We now state the main result.

Theorem 5. Let $\mathcal{U} \subset \mathcal{X}^{1}(M)$ be a $C^{1}$-open set such that, for every $X \in \mathcal{U}$ there is an open set $U$ of $M$ such that the chain recurrence class $C$ is robustly chain transitive in $U$. Then $X$ is singular volume partial hyperbolic in $U$.

\section{Basic definitions and preliminaries}

\subsection{Chain recurrent classes and filtrating neighborhoods}

The following notions and theorems are due to Conley $\mathrm{Co}$ and they can be found in several other references (for example [AN]).

- We say that pair of sequences $\left\{x_{i}\right\}_{0 \leq i \leq k}$ and $\left\{t_{i}\right\}_{0 \leq i \leq k-1}, k \geq 1$, are an $\varepsilon$-pseudo orbit from $x_{0}$ to $x_{k}$ for a flow $\phi$, if for every $0 \leq i \leq k-1$ one has

$$
t_{i} \geq 1 \text { and } d\left(x_{i+1}, \phi^{t_{i}}\left(x_{i}\right)\right)<\varepsilon \text {. }
$$

- A compact invariant set $\Lambda$ is called chain transitive if for any $\varepsilon>0$ and for any $x, y \in \Lambda$ there is an $\varepsilon$-pseudo orbit from $x$ to $y$.

- We say that $x, y \in M$ are chain related if, for every $\varepsilon>0$, there are $\varepsilon$ pseudo orbits form $x$ to $y$ and from $y$ to $x$. This is an equivalence relation.

- We say that $x \in M$ is chain recurrent if for every $\varepsilon>0$, there is an $\varepsilon$-pseudo orbit from $x$ to $x$. We call the set of chain recurrent points, the Chain recurrent set and we note it $\mathfrak{R}(M)$. The equivalent classes of this equivalence relation are called chain recurrence classes.

Definition 3. - An attracting region (also called trapping region by some authors) is a compact set $U$ so that $\phi^{t}(U)$ is contained in the interior of $U$ for every $t>0$. The maximal invariant set in an attracting region is called an attracting set. A repelling region is an attracting region for $-X$, and the maximal invariant set is called a repeller.

- A filtrating region is the intersection of an attracting region with a repelling region.

- Let $C$ be a chain recurrent class of $M$ for the flow $\phi$. A filtrating neighborhood of $C$ is a (compact) neighborhood which is a filtrating region. Co.

The following is a corollary of the fundamental theorem of dynamical systems

Corollary 6. [Co] Let $\phi$ be a $C^{1}$-vector field on a compact manifold $M$. Every chain class $C$ of $X$ admits a basis of filtrating neighborhoods, that is, every neighborhood of $C$ contains a filtrating neighborhood of $C$. 
Definition 4. Let $C$ be a Chain recurrent class of $M$ for the vector field $X$. Let $C$ be such that there is a filtrating neighborhood $U$ such that $C$ is the maximal invariant set in $U$. We say that $C$ is robustly chain transitive if there is a $C^{1}$ neighborhood of $X$ called $\mathcal{U}$ such that for every $Y \in \mathcal{U}$, the maximal invariant set for $Y\left(C_{Y}\right)$ in $U$ is a unique chain class.

Definition 5. Let $C$ be a robustly chain transitive class of $M$ for the vector field $X$. We say that $C$ is robustly transitive if there is a $C^{1}$ neighborhood of $X$ called $\mathcal{U}$ such that for every $Y \in \mathcal{U}$, there is an orbit for $Y$ which is dense in $C_{Y}$.

\section{$2.2 \quad$ Linear cocycle}

Let $\phi=\left\{\phi^{t}\right\}_{t \in \mathbb{R}}$ be a topological flow on a compact metric space $K$. A linear cocycle over $(K, \phi)$ is a continuous map $A^{t}: E \times \mathbb{R} \rightarrow E$ defined by

$$
A^{t}(x, v)=\left(\phi^{t}(x), A_{t}(x) v\right),
$$

where

- $\pi: E \rightarrow K$ is a $d$ dimensional linear bundle over $K$;

- $A_{t}:(x, t) \in K \times \mathbb{R} \mapsto G L\left(E_{x}, E_{\phi^{t}(x)}\right)$ is a continuous map that satisfies the cocycle relation :

$$
A_{t+s}(x)=A_{t}\left(\phi^{s}(x)\right) A_{s}(x), \quad \text { for any } x \in K \text { and } t, s \in \mathbb{R}
$$

Note that $\mathcal{A}=\left\{A^{t}\right\}_{t \in \mathbb{R}}$ is a flow on the space $E$ which projects on $\phi^{t}$.

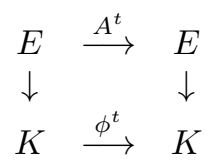

If $\Lambda \subset K$ is a $\phi$-invariant subset, then $\pi^{-1}(\Lambda) \subset E$ is $\mathcal{A}$-invariant, and we call the restriction of $\mathcal{A}$ to $\Lambda$ the restriction of $\left\{A^{t}\right\}$ to $\pi^{-1}(\Lambda)$.

\subsection{Hyperbolic structures and dominated splitting on lin- ear cocycles}

In this section we give a rough presentation of some of the hyperbolic structures over cocycles.

Definition 6. Let $\phi$ be a topological flow on a compact metric space $\Lambda$. We consider a vector bundle $\pi: E \rightarrow \Lambda$ and a linear cocycle $\mathcal{A}=\left\{A^{t}\right\}$ over $(\Lambda, X)$.

We say that $\mathcal{A}$ admits a dominated splitting over $\Lambda$ if

- there exists a splitting $E=E^{1} \oplus \cdots \oplus E^{k}$ over $\lambda$ into $k$ sub bundles 
- The dimension of the sub bundles is constant, i.e. $\operatorname{dim}\left(E_{x}^{i}\right)=\operatorname{dim}\left(E_{y}^{i}\right)$ for all $x, y \in \Lambda$ and $i \in\{1 \ldots k\}$,

- The splitting is invariant, i.e. $A^{t}(x)\left(E_{x}^{i}\right)=E_{\phi^{t}(x)}^{i}$ for all $i \in\{1 \ldots k\}$,

- there exists a $t>0$ such that for every $x \in \Lambda$ and any pair of non vanishing vectors $v \in E_{x}^{i}$ and $u \in E_{x}^{j}, i<j$ one has

$$
\frac{\left\|A^{t}(u)\right\|}{\|u\|} \leq \frac{1}{2} \frac{\left\|A^{t}(v)\right\|}{\|v\|}
$$

We denote $E^{1} \oplus_{\prec} \cdots \oplus_{\prec} E^{k}$.the splitting is $t$-dominated.

A classical result (see for instance [BDV] Appendix B]) asserts that the bundles of a dominated splitting varies continuously with the vector field in the $\mathcal{C}^{1}$ topology. A given cocycle may admit several dominated splittings. However, the dominated splitting is unique if one prescribes the dimensions $\operatorname{dim}\left(E^{i}\right)$.

We can consider in a metric spaces $K$, an invariant sub spaces $\Lambda$ of $K$ that is not compact. In this case we would ask for the norm of $\mathcal{A}$ to be bounded. Note that the dominated splitting defined as above is uniform with respect to the point. This is particularly important when we consider a dominated splitting over a set that is not compact.

One says that one of the bundle $E^{i}$ is volume contracting (resp. expanding) if there is $t>0$ so that one has

$$
\operatorname{Det}\left(J\left(\left.A^{t}\right|_{E^{i}}\right)\right)<\frac{1}{2}
$$

$\left(\right.$ resp. $\operatorname{Det}\left(J\left(\left.A^{-t}\right|_{E^{i}}\right)\right)<\frac{1}{2}$.

Definition 7. We say that the linear cocycle $\mathcal{A}$ is volume partial hyperbolic over $\Lambda$ if there is a finest dominated splitting $E=E^{1} \oplus_{\prec} \cdots \oplus E^{l}$ over $\Lambda$ is such that the extremal bundles $E^{1}$ and resp. $E^{l}$ volume contracting/expanding

\subsection{Linear Poincaré flow}

Let $X$ be a $C^{1}$ vector field on a compact manifold $M$. We denote by $\phi^{t}$ the flow of $X$.

Definition 8. The normal bundle of $X$ is the vector bundle $N_{X}$ over $M \backslash$ $\operatorname{Sing}(X)$ defined as follows: the fiber $N_{X}(x)$ of $x \in M \backslash \operatorname{Sing}(X)$ is the quotient space of $T_{x} M$ by the vector line $\mathbb{R} . X(x)$.

Note that, if $M$ is endowed with a Riemannian metric, then $N_{X}(x)$ is canonically identified with the orthogonal space of $X(x)$ :

$$
N_{X}=\{(x, v) \in T M, v \perp X(x)\}
$$

Consider $x \in M \backslash \operatorname{Sing}(M)$ and $t \in \mathbb{R}$. Thus $D \phi^{t}(x): T_{x} M \rightarrow T_{\phi^{t}(x)} M$ is a linear automorphism mapping $X(x)$ onto $X\left(\phi^{t}(x)\right)$. Therefore $D \phi^{t}(x)$ passes to the quotient as an linear automorphism $\psi^{t}(x): N_{X}(x) \rightarrow N_{X}\left(\phi^{t}(x)\right)$ : 


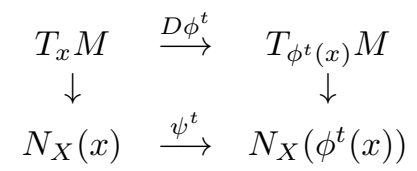

where the vertical arrow are the canonical projection of the tangent space to the normal space parallel to $X$.

Proposition 7. Let $X$ be a $C^{1}$ vector field on a manifold and $\Lambda$ be a compact invariant set of $X$. Assume that $\Lambda$ does not contained any singularity of $X$. Then $\Lambda$ is hyperbolic if and only if the linear Poincaré flow over $\Lambda$ is hyperbolic.

Notice that the notion of dominated splitting for non-singular flows is sometimes better expressed in term of Linear Poincaré flow: for instance, the linear Poincaré flow of a robustly transitive vector field always admits a dominated splitting, when the flow by itself may not admit any dominated splitting (see for instance $[\mathrm{V}]$ and $[\mathrm{D}]$ ).

\subsection{Extended linear Poincaré flow}

We are dealing with singular flows and the linear Poincare flow is not defined on the singularity of the vector field $X$. However we can include the linear Poincaré flow in a flow, called extended linear Poincaré flow defined in GLW], on a larger set.

This flow will be a linear co-cycle define on some linear bundle over a manifold, that we define now.

Definition 9. Let $M$ be a manifold of dimension $d$.

- We call the projective tangent bundle of $M$, and denote by $\Pi_{\mathbb{P}}: \mathbb{P} M \rightarrow M$, the fiber bundle whose fiber $\mathbb{P}_{x}$ is the projective space of the tangent space $T_{x} M$ : in other word, a point $L_{x} \in \mathbb{P}_{x}$ is a 1-dimensional vector subspace of $T_{x} M$.

- We call the tautological bundle of $\mathbb{P} M$, and we denote by $\Pi_{\mathcal{T}}: \mathcal{T} M \rightarrow \mathbb{P} M$, the 1-dimensional vector bundle over $\mathbb{P} M$ whose fiber $\mathcal{T}_{L}, L \in \mathbb{P} M$, is the the line $L$ itself.

- We call normal bundle of $\mathbb{P} M$ and we denote by $\Pi_{\mathcal{N}}: \mathcal{N} M \rightarrow \mathbb{P} M$, the $d$-1-dimensional vector bundle over $\mathbb{P} M$ whose fiber $\mathcal{N}_{L}$ over $L \in \mathbb{P}_{x}$ is the quotient space $T_{x} M / L$.

If we endow $M$ with riemannian metric, then $\mathcal{N}_{L}$ is identified with the orthogonal hyperplane of $L$ in $T_{x} M$.

Let $X$ be a $C^{r}$ vector field on a compact manifold $M$, and $\phi^{t}$ its flow. The natural actions of the derivative of $\phi^{t}$ on $\mathbb{P} M$ and $\mathcal{N} M$ define $C^{r-1}$ flows on these manifolds. More precisely, for any $t \in \mathbb{R}$, 
- We denote by $\phi_{\mathbb{P}}^{t}: \mathbb{P} M \rightarrow \mathbb{P} M$ the $C^{r-1}$ diffeomorphism defined by

$$
\phi_{\mathbb{P}}^{t}\left(L_{x}\right)=D \phi^{t}\left(L_{x}\right) \in \mathbb{P}_{\phi^{t}(x)} .
$$

- We denote by $\psi_{\mathcal{N}}^{t}: \mathcal{N} M \rightarrow \mathcal{N} M$ the $C^{r-1}$ diffeomorphism whose restriction to a fiber $\mathcal{N}_{L}, L \in \mathbb{P}_{x}$, is the linear automorphisms onto $\mathcal{N}_{\phi_{\mathbb{P}}^{t}(L)}$ defined as follows: $D \phi^{t}(x)$ is a linear automorphism from $T_{x} M$ to $T_{\phi^{t}(x)} M$, which maps the line $\mathcal{T}_{L} \subset T_{x} M$ onto the line $\mathcal{T}_{p h i_{\mathrm{p}}^{t}(L)}$. Therefore it passe to the quotient in the announced linear automorphism.

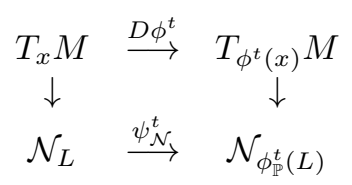

Note that $\phi_{\mathbb{P}}^{t}, t \in \mathbb{R}$ defines a flow on $\mathbb{P}_{M}$ which is a co-cycle over $\phi^{t}$ whose action on the fibers is by projective maps.

The one-parameter family $\psi_{\mathcal{N}}^{t}$ defines a flow on $\mathcal{N} M$, which is a linear cocycle over $\phi_{\mathbb{P}}^{t}$. We call $\psi_{\mathcal{N}}^{t}$ the extended linear Poncaré flow. We can summarize by the following diagrams:

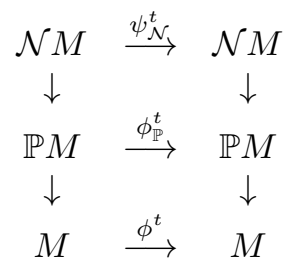

Remark 10. The extended linear Poincaré flow is really an extension of the linear Poincaré flow defined in the previous section; more precisely:

Let $S_{X}: M \backslash \operatorname{Sing}(X) \rightarrow \mathbb{P} M$ be the section of the projective bundle defined as $S_{X}(x)$ is the line $\langle X(x)\rangle \in \mathbb{P}_{x}$ generated by $X(x)$. Then $N_{X}(x)=\mathcal{N}_{S_{X}(x)}$ and the linear automorphisms $\psi^{t}: N_{X}(x) \rightarrow N_{X}\left(\phi^{t}(x)\right)$ and $\psi_{\mathcal{N}}^{t}: \mathcal{N}_{S_{X}(x)} \rightarrow$ $\mathcal{N}_{S_{X}\left(\phi^{t}(x)\right)}$

\subsection{Some classic theorems.}

We now present some results that allow us a better control of the size of the invariant manifolds near singularities. We need for this the definition of $(\eta, T, E)^{*}$ contracting orbit arcs.

Definition 11. Given $\phi_{t}$ a flow induced by $X \in \mathcal{X}^{1}(M), \Lambda$ a compact invariant set of $\phi_{t}$, and $E \subset\|\Lambda-\operatorname{Sing}(X)\|$ an invariant bundle of the linear Poincaré flow $\psi_{t}$. For $\eta>0$ and $T>0, x \in \Lambda-\operatorname{Sing}(X)$ is called $(\eta, T, E)^{*}$ contracting if for any $n \in \mathbb{N}$,

$$
\prod_{i=0}^{n-1}\left\|\psi_{T \mid E\left(\phi_{i T}(x)\right)}^{*}\right\| \leq e^{-n \eta} .
$$


Similarly $x \in \Lambda-\operatorname{Sing}(X)$ is called $(\eta, T, F)^{*}$ expanding if it is $(\eta, T, F)^{*}$ contracting for $-X$.

To find the $(\eta, T, E)^{*}$ contracting orbit arcs, one needs the classical result due to V.Pliss:

Lemma 8. [?](Pliss lemma) Given a number $A$. Let $\left\{a_{1}, \cdots, a_{n}\right\}$ be a sequence of numbers which are bounded from above by A. Assume that there exists a number $\xi<A$ such that $\sum_{i=1}^{n} a_{i} \geq n \cdot \xi$, then for any $\xi^{\prime}<\xi$, there exist $l$ integers $1 \leq t_{1}<\cdots<t_{l} \leq n$ such that

$$
\frac{1}{t_{j}-k} \sum_{i=k+1}^{t_{j}} a_{i} \geq \xi^{\prime}, \text { for any } j=1, \cdots, l \text { and any integer } k=0, \cdots t_{j}-1 \text {. }
$$

Moreover, one has the estimate $\frac{l}{n} \geq \frac{\xi-\xi^{\prime}}{A-\xi^{\prime}}$.

Remark 12. From ?? and 8 we have that if the periodic orbits of a flow are volume contracting in some bundle $E$ in the period, we can always find $(\eta, T, E)^{*}$ volume contracting points.

Lemma 9 (Connecting lemma). [BC]. Given $\phi_{t}$ induced by a vector field $X \in \mathcal{X}^{1}(M)$ such that all periodic orbits of $X$ are hyperbolic. For any $C^{1}$ neighborhood $\mathcal{U}$ of $X$ and $x, y \in M$ if $y$ is chain attainable from $x$, then there exists $Y \in U$ and $t>0$ such that $\phi_{t}^{Y}(x)=y$. Moreover the following holds: For any $k \geq 1$, let $\left\{x_{i, k}, t_{i, k}\right\}_{i=0}^{n_{k}}$ be an $(1 / k, T)$-pseudo orbit from $x$ to $y$ and denote by

$$
\Delta_{k}=\bigcup_{i=0}^{n_{k}-1} \phi_{\left[0, t_{i, k}\right]}\left(x_{i, k}\right) .
$$

Let $\Delta$ be the upper Hausdorff limit of $\Delta_{k}$. Then for any neighborhood $U$ of $\Delta$, there exists $Y \in U$ with $Y=X$ on $M \backslash U$ and $t>0$ such that $\phi_{t}^{Y}(x)=y$.

For a generic vector field $X \in \mathcal{X}^{1}(M)$ we have:

Theorem 10. [C] There exists a $G_{\text {approx }} \subset \mathcal{X}^{1}(M)$ a generic set such that for every $X \in G_{\text {approx }}$ and for every $C$ a chain recurrence class there exists a sequence of periodic orbits $\gamma_{n}$ which converges to $C$ in the Hausdorff topology.

The following theorem by Mañe was first introduced in $\mathrm{Ma}$ and it was used in Ma2 to prove the stability conjecture. The idea behind this theorem is that the lack of hyperbolicity in a set can be detected by the clack of hyperbolicity in a periodic orbit of a $C^{1}$ close system.

Definition 13. Let $f$ be a diffeomorphism of a compact manifold $M$ with a Riemmanian metric $d$. A point $x$ is well closable if for every $\epsilon$ there are diffeomorphisms $g$, that are $\epsilon-C^{1}$ close to $f$ and periodic points $y$ for $g$ with period $T_{y}$, such that

$$
d\left(f^{i}(x), g^{i}(y)\right)<\epsilon \text { for all } 0 \leq i \leq T_{y} .
$$

We note the set of well closable points of $f$ as $\mathcal{W}(f)$ 
Theorem (Ergodic closing lemma). Let $f$ be a diffeomorphism and $\mu$ an $f$-invariant probability measure, then almost every point is well closable. That is

$$
\mu \mathcal{W}(f)=1 \text {. }
$$

The version of this theorem for flows is almost the same with one exception: the well closable points might be closed by a singularity.

Definition 14. Let $X$ be a vector field of a compact manifold $M$ with a Riemannian metric $d$, and $\phi$ its associated flow. A point $x$ is well closable if for every $\epsilon$ there are vector fields $Y$, that are $\epsilon-C^{1}$ close to $X$ and critical elements (closed orbits) $y$ for $Y$ with period $T_{y}$, such that

$$
d\left(\phi_{t}^{X}(x), \phi_{t}^{Y}(y)\right)<\epsilon \text { for all } 0 \leq t \leq T_{y} .
$$

We note the set of well closable points of $X$ as $\mathcal{W}(X)$

Theorem (Ergodic closing lemma for flows). Let $X$ be a vector field of a compact manifold $M$ with a Riemannian metric $d$. For every $T>0$ and $\mu$ a $\phi_{T}$-invariant probability measure, almost every point is well closable. That is

$$
\mu \mathcal{W}(f)=1 \text {. }
$$

\section{Singular volume partial hyperbolicity}

\subsection{Strong stable, strong unstable and center spaces asso- ciated to a hyperbolic singularity.}

Let $X$ be a vector field and $\sigma \in \operatorname{Sing}(X)$ be a hyperbolic singular point of $X$. Let $\lambda_{k}^{s} \ldots \lambda_{2}^{s}<\lambda_{1}^{s}<0<\lambda_{1}^{u}<\lambda_{2}^{u} \ldots \lambda_{l}^{u}$ be the Lyapunov exponents of $\phi_{t}$ at $\sigma$ and let $E_{k}^{s} \oplus_{<} \cdots E_{2}^{s} \oplus_{<} E_{1}^{s} \oplus_{<} E_{1}^{u} \oplus_{<} E_{2}^{u} \oplus_{<} \cdots \oplus_{<} E_{l}^{s}$ be the corresponding (finest) dominated splitting over $\sigma$.

A subspace $F$ of $T_{\sigma} M$ is called a center subspace if it is of one of the possible form below:

- Either $F=E_{i}^{s} \oplus_{<} \cdots E_{2}^{s} \oplus_{<} E_{1}^{s}$

- Or $F=E_{1}^{u} \oplus_{<} E_{2}^{u} \oplus_{<} \cdots \oplus_{<} E_{j}^{s}$

- Or else $F=E_{k}^{s} \oplus_{<} \cdots E_{2}^{s} \oplus_{<} E_{1}^{s} \oplus_{<} E_{1}^{u} \oplus_{<} E_{2}^{u} \oplus_{<} \cdots \oplus_{<} E_{l}^{s}$

A subspace of $T_{\sigma} M$ is called a strong stable space, and we denote it $E_{i}^{s s}(\sigma)$, if there in $i \in\{1, \ldots, k\}$ such that:

$$
E_{i}^{s s}(\sigma)=E_{k}^{s} \oplus_{<} \cdots E_{j+1}^{s} \oplus_{<} E_{i}^{s}
$$

A classical result from hyperbolic dynamics asserts that for any $i$ there is a unique injectively immersed manifold $W_{i}^{s s}(\sigma)$, called a strong stable manifold tangent at $E_{i}^{s s}(\sigma)$ and invariant by the flow of $X$.

We define analogously the strong unstable spaces $E_{j}^{u u}(\sigma)$ and the strong unstable manifolds $W_{j}^{u u}(\sigma)$ for $j=1, \ldots, l$. 


\subsection{The lifted maximal invariant set and the singular points}

Let $\Lambda$ be a maximal invariant set for a flow $X$. We define de lifted maximal invariant set, that is:

$$
\Lambda_{\mathbb{P}, U}(X)=\overline{\{<X(x)>\in \mathbb{P} M \text { such that } x \in \Lambda\}} .
$$

The lifted maximal invariant set does not vary upper semi-continuously with $X$.

Let $U$ be a compact region, and $X$ a vector field. Let $\sigma \in \operatorname{Sing}(X) \cap U$ be a hyperbolic singularity of $X$ contained in $U$. We are interested on $\Lambda_{\mathbb{P}, U}(X) \cap \mathbb{P}_{\sigma}$. As in $\mathrm{BdL}$ the aim of this section is to add to the lifted maximal invariant set $\Lambda_{\mathbb{P}, U}$, some set over the singular points in order to recover some upper semicontinuity properties.

\subsubsection{Upper semi continuity of the lift}

We can consider now the smallest set that varies upper semi continuously with the vector field that was introduced in GLW]. The set is defined as follows:

Definition 15. Let $U$ be a compact region and $X$ a $C^{1}$ vector field. Let $\mathcal{U}$ be a neighborhood of $X$ Then we define

$$
\widetilde{\Lambda}=\overline{\{<Y(x)>\in \mathbb{P} M \text { such that } x \in U \cap \operatorname{Per}(Y) \text { and } Y \in \mathcal{U}\}} .
$$

When the hypothesis of our problem gives us information about an open set of vector fields, for instance when we are talking about a robustly transitive sets, this lifted set of directions prooves very easy to work with. However, when we intend to define a dominated splitting structure or a partial hyperbolic structure over it, then we want to do it over a set that one can detect by looking at only one vector field. Therefore in $[\mathrm{BdL}$ a bigger set is introduced, that does not relay on information of the perturbations of the flow and varies upper semi continuously with the vector field.

\subsection{The extended maximal invariant set}

We define the escaping stable space $E_{\sigma, U}^{s s}$ as the biggest strong stable space $E_{j}^{s s}(\sigma)$ such that the corresponding strong stable manifold $W_{j}^{s s}(\sigma)$ is escaping, that is:

$$
\Lambda_{X, U} \cap W_{j}^{s s}(\sigma)=\{\sigma\} .
$$

We define the escaping unstable space analogously.

We define the central space $E_{\sigma, U}^{c}$ of $\sigma$ in $U$ the center space such that

$$
T_{\sigma} M=E_{\sigma, U}^{s s} \oplus E_{\sigma, U}^{c} \oplus E_{\sigma, U}^{u u}
$$

We denote by $\mathbb{P}_{\sigma, U}^{i}$ the projective space of $E^{i}(\sigma, U)$ where $i \in\{s s, u u, c\}$. The proofs of all lemmas proppositions and theorems in this subsection can be found in $\mathrm{BdL}$ 
Lemma 11. The central space $E_{\sigma, U}^{c}$ is the smallest center space containing $\Lambda_{\mathbb{P}, U} \cap \mathbb{P}_{\sigma}$.

Lemma 12. Let $U$ be a compact region. Let $\sigma$ be a hyperbolic singular point in $U$, that has a continuation $\sigma_{Y}$ for vector fields $Y$ in a $C^{1}$-neighborhood of $X$. Then both escaping strong stable and unstable spaces $E_{\sigma_{Y}, U}^{s s}$ and $E_{\sigma_{Y}, U}^{u u}$ depend lower semi-continuously on $Y$.

As a consequence the central space $E_{\sigma_{Y}, U}^{c}$ of $\sigma_{Y}$ in $U$ for $Y$ depends upper semi-continuously on $Y$, and the same happens for its projective space $\mathbb{P}_{\sigma_{Y}, U}^{s}$.

Definition 16. Let $U$ be a compact region and $X$ a vector field whose singular points are hyperbolic. Then the set

$$
B(X, U)=\Lambda_{\mathbb{P}, U} \cup \bigcup_{\sigma \in \operatorname{Sing}(X) \cap U} \mathbb{P}_{\sigma, U}^{c} \subset \mathbb{P} M
$$

is called the extended maximal invariant set of $X$ in $U$

Proposition 13. Let $U$ be a compact region and $X$ a vector field whose singular points are hyperbolic. Then the extended maximal invariant set $B(X, U)$ of $X$ in $U$ is a compact subset of $\mathbb{P} M$, invariant under the flow $\phi_{\mathbb{P}}^{t}$. Furthermore, the map $X \mapsto B(X, U)$ depends upper semi-continuously on $X$.

Proposition 14. Let $U$ be a compact region and $X$ a vector field whose singular points are hyperbolic. Then the extended maximal invariant set $B(X, U)$ of $X$ in $U$ is a compact subset of $\mathbb{P} M$, invariant under the flow $\phi_{\mathbb{P}}^{t}$. Furthermore, the map $X \mapsto B(X, U)$ depends upper semi-continuously on $X$.

\subsection{Extending chain recurrence classes}

Conley theory asserts that any chain recurrent $C$ class admits a basis of neighborhood which are nested filtrating neighborhood $U_{n+1} \subset U_{n}, C=\bigcap U_{n}$ We define

$$
\widetilde{\Lambda(C)}=\bigcap_{n} \Lambda \widetilde{\left(X, U_{n}\right)} \text { and } B(C)=\bigcap_{n} B\left(X, U_{n}\right) .
$$

These two sets are independent of the choice of the sequence $U_{n}$ and $\widetilde{\Lambda(C)} \subset$ $B(C)$.

Definition 17. We say that a chain recurrence class $C$ has a given singular hyperbolic structure if $\widetilde{\Lambda(C)}$ carries this singular hyperbolic structure.

If $\sigma \in \operatorname{Sing}(X)$ is an hyperbolic singular point we define $E_{\sigma}^{c}=\bigcap_{n} E_{\sigma, U_{n}}^{c}$ and we call it the center space of $\sigma$. We denote $\mathbb{P}_{\sigma}^{c}=\mathbb{P} E_{\sigma}^{c}$, its projective space.

Remark 18. Consider the open and dense set of vector fields whose singular points are all hyperbolic. In this open set the singularities depend continuously on the field. Then for every singular point $\sigma$, the projective center space $\mathbb{P}_{\sigma}^{c}$ 
varies upper semi continuously, and in particular the dimension $\operatorname{dim} E_{\sigma}^{c}$ varies upper semi-continuously. As it is a non-negative integer, it is locally minimal and locally constant on a open and dense subset.

We will say that such a singular point has locally minimal center space.

\subsection{Reparametrizations}

Let $\mathcal{A}=\left\{A^{t}(x)\right\}$ and $\mathcal{B}=\left\{B^{t}(x)\right\}$ be two linear cocycles on the same linear bundle $\pi: \mathcal{E} \rightarrow \Lambda$ and over the same flow $\phi^{t}$ on a compact invariant set $\Lambda$ of a manifold $M$. We say that $\mathcal{B}$ is a reparametrization of $\mathcal{A}$ if there is a continuous map $h=\left\{h^{t}\right\}: \Lambda \times \mathbb{R} \rightarrow(0,+\infty)$ so that for every $x \in \Lambda$ and $t \in \mathbb{R}$ one has

$$
B^{t}(x)=h^{t}(x) A^{t}(x) .
$$

The reparametrizing map $h^{t}$ satisfies the cocycle relation

$$
h^{r+s}(x)=h^{r}(x) h^{s}\left(\phi^{r}(x)\right),
$$

and is called a cocycle.

One easily check the following lemma:

Lemma 15. Let $\mathcal{A}$ be a linear cocycle and $\mathcal{B}$ be a reparametrization of $\mathcal{A}$. Then any dominated splitting for $\mathcal{A}$ is a dominated splitting for $\mathcal{B}$.

Remark 19. - If $h^{t}$ is a cocycle, then for any $\alpha \in \mathbb{R}$ the power $\left(h^{t}\right)^{\alpha}: x \mapsto$ $\left(h^{t}(x)\right)^{\alpha}$ is a cocycle.

- If $f^{t}$ and $g^{t}$ are cocycles then $h^{t}=f^{t} \cdot g^{t}$ is a cocycle.

A cocycle $h^{t}$ is called a coboundary if there is a continuous function $h: \Lambda \rightarrow$ $(0,+\infty)$ so that

$$
h^{t}(x)=\frac{h\left(\phi^{t}(x)\right)}{h(x)} .
$$

A coboundary cocycle in uniformly bounded. Two cocycles $g^{t}, h^{t}$ are called cohomological if $\frac{g^{t}}{h^{t}}$ is a coboundary.

Remark 20. The cohomological relation is an equivalence relation among the cocycle and is compatible with the product: if $g_{1}^{t}$ and $g_{2}^{t}$ are cohomological and $h_{1}^{t}$ and $h_{2}^{t}$ are cohomological then $g_{1}^{t} h_{1}^{t}$ and $g_{2}^{t} h_{2}^{t}$ are cohomological.

Lemma 16. if $g$ and $h$ are cohomological then $g \cdot \mathcal{A}$ is hyperbolic if and only if $h \cdot \mathcal{A}$ is hyperbolic. 


\subsubsection{Reparametrizing cocycle associated to a singular point}

Let $X$ be a $C^{1}$ vector field, $\phi^{t}$ its flows, and $\sigma$ be a hyperbolic singularity of $X$. We denote by $\Lambda_{X} \subset \mathbb{P} M$ the union

$$
\Lambda_{X}=\overline{\{\mathbb{R} X(x), x \notin \operatorname{Sing}(X)\}} \cup \bigcup_{x \in \operatorname{Sing}(X)} \mathbb{P} T_{x} M .
$$

It can be shone easily that this set is upper semi-continuous, as in the case of $B(X, U)$ (see 14)

Lemma 17. $\Lambda_{X}$ is a compact subset of $\mathbb{P} M$ invariant under the flow $\phi_{\mathbb{P}}^{t}$, and the map $X \mapsto \Lambda_{X}$ is upper semi-continuous. Finally, if the singularities of $X$ are hyperbolic then, for any compact regions one has $B(X, U) \subset \Lambda_{X}$.

Let $U_{\sigma}$ be a compact neighborhood of $\sigma$ on which $\sigma$ is the maximal invariant.

Let $V_{\sigma}$ be a compact neighborhood of $\operatorname{Sing}(X) \backslash\{\sigma\}$ so that $V_{\sigma} \cap U_{\sigma}=\emptyset$. We fix a $\left(C^{1}\right)$ Riemmann metric $\|\cdot\|$ on $M$ so that

$$
\|X(x)\|=1 \text { for all } x \in M \backslash\left(U_{\sigma} \cup V_{\sigma}\right) .
$$

Consider the map $h: \Lambda_{X} \times \mathbb{R} \rightarrow(0,+\infty), h(L, t)=h^{t}(L)$, defined as follows:

- if $L \in \mathbb{P} T_{x} M$ with $x \notin U_{\sigma}$ and $\phi^{t}(x) \notin U_{\sigma}$, then $h^{t}(L)=1$;

- if $L \in \mathbb{P} T_{x} M$ with $x \in U_{\sigma}$ and $\phi^{t}(x) \notin U_{\sigma}$ then $L=\mathbb{R} X(x)$ and $h^{t}(L)=$ $\frac{1}{\|X(x)\|}$;

- if $L \in \mathbb{P} T_{x} M$ with $x \notin U_{\sigma}$ and $\phi^{t}(x) \in U_{\sigma}$ then $L=\mathbb{R} X(x)$ and $h^{t}(L)=$ $\left\|X\left(\phi^{t}(x)\right)\right\|$;

- if $L \in \mathbb{P} T_{x} M$ with $x \in U_{\sigma}$ and $\phi^{t}(x) \in U_{\sigma}$ but $x \neq \sigma$ then $L=\mathbb{R} X(x)$ and $h^{t}(L)=\frac{\| X\left(\phi^{t}(x) \|\right.}{\|X(x)\|}$;

- if $L \in \mathbb{P} T_{\sigma} M$ then $h^{t}(L)=\frac{\left\|\phi_{\mathrm{P}}^{t}(u)\right\|}{\|u\|}$ where $u$ is a vector in $L$.

Note that the case in which $x$ is not the singularity and $x \in U_{\sigma}$, then $h$ can be written as in the last item, taking $u=X(x)$.

As it was proven in $\mathrm{BdL}$ the map we just defined is a (continuous) cocycle on $\Lambda_{X}$ and it's cohomology class, is independent from the choice of the metric $\|$.$\| and of the neighborhoods.$

Also from $\mathrm{BdL}$ we have :

Lemma 18. Consider a vector field $X$ and a hyperbolic singularity $\sigma$ of $X$. Then there is a $C^{1}$-neighborhood $\mathcal{U}$ of $X$ so that $\sigma$ has a well defined hyperbolic continuation $\sigma_{Y}$ for $Y$ in $\mathcal{U}$ and for any $Y \in \mathcal{U}$ there is a map $h_{Y}: \Lambda_{Y} \times \mathbb{R} \rightarrow$ $(0,+\infty)$ so that

- for any $Y, h_{Y}$ is a cocycle belonging to the cohomology class $\left[h\left(Y, \sigma_{Y}\right)\right]$

- $h_{Y}$ depends continuously on $Y:$ if $Y_{n} \in \mathcal{U}$ converge to $Z \in \mathcal{U}$ for the $C^{1}$ topology and if $L_{n} \in \Lambda_{Y_{n}}$ converge to $L \in \Lambda_{Z}$ then $h_{Y_{n}}^{t}\left(L_{n}\right)$ tends to $h_{Z}^{t}(L)$ for every $t \in \mathbb{R}$; furthermore this convergence is uniform in $t \in[-1,1]$. 


\section{Definition of singular volume partial hyper- bolicity}

Let $X$ be a vector field with a dominated splitting of the linear Poincaré flow

$$
\mathcal{N}_{L}=\mathcal{N}^{s} \oplus \cdots \oplus \mathcal{N}^{u}
$$

where $\mathcal{N}_{L}^{s}$ and $\mathcal{N}_{L}^{u}$ denote the extremal bundles, over a chainrecurrence class $C$. Supose as well that the set $S$ of the singularities in $C$ contains only hyperbolic singularities. We subdivide $S$ as follows

- the set $S_{E c}$ of singular points whose escaping stable space has dimension smaller than $\mathcal{N}_{L}^{s}$,

- the set $S_{E}$ of singular points whose escaping stable space has dimension bigger or equal than $\mathcal{N}_{L}^{s}$,

- the set $S_{F c}$ of singular points whose escaping unstable space has dimension smaller than $\mathcal{N}_{L}^{u}$,

- the set $S_{F}$ of singular points whose escaping unstable space has dimension bigger or equal than $\mathcal{N}_{L}^{u}$.

Definition 21. Let $X$ be a $C^{1}$-vector field on a compact manifold and let $C$ bea chain recurrence class. We denote $S=\operatorname{Sing}(X) \cap C$. One says that $X$ is singular volume partial hyperbolic on $C$ if

1. The restriction of the extended linear Poincaré flow $\left\{\psi_{\mathcal{N}}^{t}\right\}$ to the extended maximal invariant set $B(C)$ admits a finest dominated splitting

$$
\mathcal{N}_{L}=\mathcal{N}^{s} \oplus \cdots \oplus \mathcal{N}^{u}
$$

where $\mathcal{N}_{L}^{s}$ and $\mathcal{N}_{L}^{u}$ denote the extremal bundles, and $n_{s}$ and $n_{u}$ their respective dimensions.

2. There is a subset $S_{E c} \subset S$ so that the reparametrized cocycle $h_{E c}^{t} \psi_{\mathcal{N}}^{t}$ contracts volume in restriction to the bundles $\mathcal{N}_{L}^{s}$ over $B(C)$ where $h_{E c}$ denotes

$$
h_{E c}=\Pi_{\sigma \in S_{E c}} h_{\sigma}^{n_{s}} .
$$

3. There is a subset $S_{F c} \subset S$ so that the reparametrized cocycle $h_{F c}^{t} \psi_{\mathcal{N}}^{t}$ expands volume in restriction to the bundle $\mathcal{N}_{L}^{u}$ over $B(C)$ where $h_{F c}$ denotes

$$
h_{F c}=\Pi_{\sigma \in S_{F c}} h_{\sigma}^{n_{u}} .
$$

Remark 22. If $C$ is a chain recurrence class which is singular volume hyperbolic then $X$ is singular volume hyperbolic on a small filtrating neighborhood of $C$. 
Definition 23. Let $X$ be a $C^{1}$-vector field on a compact manifold and let $\Lambda$ be a maximal invariant set in $U$. We denote $S=\operatorname{Sing}(X) \cap U$. One says that $X$ is singular volume partial hyperbolic over $\widetilde{\Lambda}(X, U)$ if

1. The restriction of the extended linear Poincaré flow $\left\{\psi_{\mathcal{N}}^{t}\right\}$ to the extended maximal invariant set $\widetilde{\Lambda}(X, U)$ admits a finest dominated splitting $\mathcal{N}_{L}=$ $\mathcal{N}^{s} \oplus \cdots \oplus \mathcal{N}^{u}$ where $\mathcal{N}_{L}^{s}$ and $\mathcal{N}_{L}^{u}$ denote the extremal bundles.

2. There is a subset $S_{E c} \subset S$ so that the reparametrized cocycle $h_{E c}^{t} \psi_{\mathcal{N}}^{t}$ contracts volume in restriction to the bundles $\mathcal{N}_{L}^{s}$ over $\widetilde{\Lambda}(X, U)$ where $h_{E c}$ denotes

$$
h_{E c}=\Pi_{\sigma \in S_{E c}} h_{\sigma}^{n_{s}} .
$$

3. There is a subset $S_{F c} \subset S$ so that the reparametrized cocycle $h_{F c}^{t} \psi_{\mathcal{N}}^{t}$ expands volume in restriction to the bundle $\mathcal{N}_{L}^{u}$ over $\widetilde{\Lambda}(X, U)$ where $h_{F c}$ denotes

$$
h_{F c}=\Pi_{\sigma \in S_{F c}} h_{\sigma}^{n_{u}} .
$$

Remark 24. If $C$ is a robustly chain transitive calss it follows that there is a neighborhood $U$ of $C$ such that $\widetilde{\Lambda}(X, U)=\widetilde{\Lambda(C)}$.

In $\overline{\mathrm{BdL}}$ it is shonw that the hyperbolic structures of $\widetilde{\Lambda(C)}$ extend to $B(C)$. This is fundamental to our purpose, since in our scenario, dealing with $\widetilde{\Lambda(C)}$ is much more convinient.

Theorem 19 (BdL). Let $X$ be a vector field on a closed manifold, whose singular points are all hyperbolic and with locally minimal center spaces. Then for every $\sigma \in \operatorname{Sing} X$, a singular volume hyperbolic structure on $\widetilde{\Lambda(C)}$ extends to $B(C)$.

Remark 25. From remark 24 and theorem 19 we get that if $C$ is robustly chain transitive, then for $X$ under the hypothesis of the theorem the definitions 21 and 23 are in fact equivalent.

\section{Analysis of the hyperbolicity in the closed curves}

In this section we prove that an open and dense subset of the vector fields having a robustly chain transitive chain recurrence class $C$ has a dominated splitting over $B(C)$. A dominated splitting over $\widetilde{\Lambda(C)}$, from $[\mathrm{BdL}$, and since for an isolated neighborhood of $C, U$, we have that $\widetilde{\Lambda(C)}=\widetilde{\Lambda}(X, U)$. Consequently we just need to find a dominated splitting over $\widetilde{\Lambda}(X, U)$ which is a rather straight forwards consequence of already existing results. 


\subsection{The set of periodic orbits}

In a series of papers such as [DPU] [BDP] $\mathrm{BB}$, and [BGV] it is shown that if a set of periodic orbits, that has periodic orbits of arbitrarily long periods, does not have a dominated splitting, then there is a perturbation of the flow having a an infinite number of sinks and sources. We state two of this result below:

Lemma 20 ( BGV Theorem 2.2). Let $\mathcal{A}$ be a bounded linear cocycle over $\pi: E \rightarrow \sum$ where $\sum$ is a set of periodic orbits, containing periodic orbits of arbitrarily long periods. Then if $\mathcal{A}$ does not admit any dominated splitting, then there exist a perturbation $\mathcal{B}$ of $\mathcal{A}$ such that $E$ is contracted or expanded by $\mathcal{B}$ along the orbit.

Lemma 21 ( $\mathrm{BDP}]$ lemma 6.1). Let $\mathcal{A}$ be a bounded linear cocycle over $\pi: E \rightarrow$ $\sum$ where $\sum$ is a set of periodic orbits, containing periodic orbits of arbitrarily long periods. Let $T(x)$ denote the period of $x \in \sum$ Suppose that $\mathcal{A}$

- $\mathcal{A}$ admits a dominated splitting $E=F_{1} \oplus \prec F_{2}$,

- $\mathcal{A}$ does not admit a dominated splitting of $F_{1}$.

- There exist a point $p$ such that $\operatorname{det}\left(\left.A^{T(p)}\right|_{F_{1}}(p)\right)>1$

then there exist a perturbation $\mathcal{B}$ of $\mathcal{A}$ and $q$ in $\sum$ such that all eigen values of $\left.A^{T(q)}\right|_{F_{1}}(q)$ are positive.

Remark 26. Both of these theorems hold if we consider the linear Poincaré flow as the cocycle, and the time $t$ of the flow as the diffeomorphism. Therefore the set of periodic orbits in a robustly chain transitive set $C$ has dominated splitting and the finest possible of this dominated splittings is such that the extremal bundles contract and expand volume.

Lemma 22. There is an open and dense subset of the vector fields $X \in \mathcal{X}^{1} M$ with a robustly chain transitive set $C$ t in a filtrating neighborhood $U \subset M$ such that set $\widetilde{\Lambda}(X, U)$ admits a finest dominated splitting of the normal bundle, for the extended linear Poincaré flow.

Proof. Take $X$ a vector field in the hypothesis of theorem 10 we can find a sequence of vector fields $Y_{n}$ converging to $X$ such that the set $C$ is the hausdorff limit of the periodic orbits of the $Y_{n}$. Since $C$ is robustly chain transitive this periodic orbits must be related, so for $Y_{n}, C$ is the closure of $U \cap \operatorname{Per}\left(Y_{n}\right)$. From lemma 20 the set $\left\{\left\langle Y_{n}(x)\right\rangle \in \mathbb{P} M\right.$ such that $\left.x \in \Lambda\right\}$ has a uniform finest dominated splitting for the extended linear Poincaré flow (note that the projection to $M$ here is one to one). Therefore, the extended linear Poincaré flow has dominated splitting over the set

$$
\overline{\left\{<Y_{n}(x)>\in \mathbb{P} M \text { such that } x \in C\right\}},
$$

since a uniform dominated splitting extends to the closure. 
Using again 10 the sequence $Y_{n}$ can be taken so that the hausdorff limit of

$$
\overline{\left\{<Y_{n}(x)>\in \mathbb{P} M \text { such that } x \in C\right\}},
$$

is exactly the set $\widetilde{\Lambda(X, U)}$, since the upper limit coincides with the Hausdorff

limit if ti exist, therefore we get that $\widetilde{\Lambda(X, U)}$ has a dominated splitting of the same dimension, for the extended linear Poincaré flow of $X$

\section{Analyzing the singulatiries}

The main problem we need to deal with now, is the distortion of the contraction and expansion rates that occurs when the periodic orbits approach the singularities. For this we will use again the reparametrized linear Poincaré flow.

With this tool, the main work in this section will be to find out which singularities need to be reparametrized. After this and following mainly BDP, we show that all closed orbits in an open set of vector fields have the structure desired.

Then, in the next section we follow the classical strategy. We use the ergodic closing lemma, to argue that if the robust chain transitive set did not have the desired structure, then there must be a critical element in a perturbed vector field that does not have that structure either. This contradiction, gives us our main theorem 5

\subsection{Center space of the singularities and the dominated splitting on $\widetilde{\Lambda}(X, U)$}

Let us consider a singularity $\sigma \in C$. We consider the following splitting of its tangent space:

$$
E^{s s} \oplus E^{c} \oplus E^{u u},
$$

noting the stable escaping, the unstable escaping and the center spaces. We suppose the singularities to be hyperbolic

Recall that from lemma 22 we have that there is a finest dominated splitting for the extended linear Poincaré flow, over $\widetilde{\Lambda}(X, U)$. We note this as follows

$$
\mathcal{N}_{L}=\mathcal{N}^{s} \oplus \mathcal{N}^{1} \oplus \cdots \oplus \mathcal{N}^{k} \oplus \mathcal{N}^{u},
$$

where $L$ is a direction in $\widetilde{\Lambda}(X, U)$.

We call $\pi_{L}: T_{x} M \rightarrow \mathcal{N}_{L}$ where $L \in \mathbb{P}_{x} M$ the projection over the normal space at a given direction $L$.

The next lemma from $\mathrm{BdL}$ tells us that there is a relation between the splitting in the singularities into escaping and center spaces, and the dominated splitting of the class for the linear Poincaré flow.

Lemma $23(\mathrm{BdL})$. Let us consider the set of vector fields $\mathcal{V}$ such that evey vector field $X \in \mathcal{V}$ has a singular chain class $C_{\sigma}$ with the following properties. We denote $S=\operatorname{Sing}(X) \cap C_{\sigma}$ 
- every $\sigma \in S$ that is hyperbolic,

- the dimension of the central space of $\sigma \in S$ is locally constant,

- the extended linear Poincaré flow over $\widetilde{\Lambda}(C(\sigma))$ has a dominated splitting,

$$
\mathcal{N}_{L}=\mathcal{N}^{E} \oplus \mathcal{N}^{F},
$$

where $L$ is any direction in $\widetilde{\Lambda}(C(\sigma))$.

Let $L$ be a direction in $\widetilde{\Lambda} \cap \mathbb{P}_{\sigma} M$, Then there is an open and dense subset of $\mathcal{V}$ noted $\mathcal{U} \subset \mathcal{V}$ such that for every $X \in \mathcal{U}$,

$$
\pi_{L}\left(E_{\sigma}^{c}\right) \subset \mathcal{N}_{L}^{E}
$$

or

$$
\pi_{L}\left(E_{\sigma}^{c}\right) \subset \mathcal{N}_{L}^{F}
$$

The next corollary is a direct consequence of the previous lemma.

Corollary 24. Let us consider the set of vector fields $\mathcal{V}$ such that evey vector field $X \in \mathcal{V}$ has a singular chain class $C_{\sigma}$ such that if we note $S=\operatorname{Sing}(X) \cap C_{\sigma}$

- every $\sigma \in S$ that is hyperbolic,

- the dimension of the central space of $\sigma \in S$ is locally constant,

- the extended linear Poincaré flow over $\widetilde{\Lambda}(C(\sigma))$ has a finest dominated splitting

$$
\mathcal{N}_{L}=\mathcal{N}^{s} \oplus \mathcal{N}^{1} \oplus \cdots \oplus \mathcal{N}^{k} \oplus \mathcal{N}^{u},
$$

Let $L$ be a direction in $\widetilde{\Lambda}(X, U) \cap \mathbb{P}_{\sigma} M$, and such that $L=<u>$. Then there is an open and dense subset of $\mathcal{V}$ noted $\mathcal{U} \subset \mathcal{V}$ such that for every $X \in \mathcal{U}$,

$$
\pi_{L}\left(E_{\sigma}^{c}\right) \cap \mathcal{N}_{L}^{s}=\emptyset,
$$

or

$$
\pi_{L}\left(E_{s 1} \oplus \cdots \oplus E_{s k} \oplus E_{u 1}\right) \subset \mathcal{N}_{L}^{s} .
$$

Now we can see a more precise definition of the sets in definition 21.

Definition 27. Let $X$ be a $C^{1}$ vector field, such that there is an open set $U$ such the maximal invariant set in $U$ is a robustly chain transitive chain recurrence class. Let us consider the finest dominated splitting for the set $\widetilde{\Lambda}(X, U)$,

$$
\mathcal{N}_{L}=\mathcal{N}_{L}^{s} \oplus \cdots \oplus \mathcal{N}_{L}^{U} .
$$

We define $S_{E c}$ the set of singularities

$$
S_{E c}=\left\{\sigma \in \operatorname{Sing}(X) \cap U \text { such that } \operatorname{dim}\left(\mathcal{N}_{L}^{s}\right)>E_{\sigma}^{s s}\right\} .
$$

Similarly we define the set $S_{F c}$ the set of singularities

$$
S_{F c}=\left\{\sigma \in \operatorname{Sing}(X) \cap U \text { such that } \operatorname{dim}\left(\mathcal{N}_{L}^{u}\right)>E_{\sigma}^{u u}\right\} .
$$

Remark 28. This definition makes the set in definition 21 to de uniquely defined and disjoint 


\subsection{Volume contraction at the singularities.}

Recall that for a hyperbolic singularity we note

$$
T_{\sigma} M=E^{s s} \oplus E^{c} \oplus E^{u u},
$$

noting the stable escaping, the unstable escaping and the center spaces. we write the center space as:

$$
E_{\sigma}^{c}=E_{s 1} \oplus \cdots \oplus E_{s k} \oplus E_{u 1} \oplus \cdots \oplus E_{u l}
$$

Lemma 25. Let $\sigma$ be a singularity of $C$, a robustly transitive chain recurrence class with an isolating filtrating neighborhood $U$. Let $\Gamma=\operatorname{Orb}(x)$ be a homoclinic orbit associated to $\sigma$. Assume as well that:

- There exists a sequence of vector fields $X_{n}$ converging to $X$ in the $C^{1}$ topology

- There exist a sequence of periodic orbit $\gamma_{n}$ of $X_{n}$ such that $\gamma_{n}$ converges to $\Gamma$ in the Hausdorff topology.

- There is a finest dominated splitting over $\widetilde{\Lambda}(X, U)$,

$$
\mathcal{N}_{L}=\mathcal{N}^{s} \oplus \mathcal{N}^{1} \oplus \cdots \oplus \mathcal{N}^{k} \oplus \mathcal{N}^{u}
$$

where $L \in \widetilde{\Lambda}(X, U)$. We note $\operatorname{dim}\left(\mathcal{N}^{u}\right)=h$ and we have that $\operatorname{dim}\left(\mathcal{N}^{s}\right)=$ $n$.

Then there is a space $E \subset T_{\sigma} M$ such that $E$ contracts volume and has dimension $n+1$, and $a F \subset T_{\sigma} M$ such that $F$ expands volume and has dimension $h+1$

Proof. Let us recall that from remark 26 the splitting

$$
\mathcal{N}_{L}=\mathcal{N}^{s} \oplus \mathcal{N}^{1} \oplus \cdots \oplus \mathcal{N}^{k} \oplus \mathcal{N}^{u}
$$

where $L$ is a direction over the set of periodic orbits of $C$ is Volume partial hyperbolic. This means that $\mathcal{N}^{s}$ contracts volume and $\mathcal{N}^{u}$ expand volume.

Since, for any $x_{n}$ in $\gamma_{n}, X_{n}\left(x_{n}\right)$ does not contract or expand at the period, then $\mathcal{N}_{L}^{s} \oplus<X_{n}\left(x_{n}\right)>$ contracts volume and $\left\langle X_{n}\left(x_{n}\right)>\oplus \mathcal{N}_{L}^{u}\right.$ expands volume uniformly at the period. Since $\gamma_{n}$ tends to the homoclinic loop $\Gamma$, their periods must tend to infinity with $n$. For $n$ large enough, and from the contraction of volume we have there exist some constants $\nu$ and $T$

$$
\prod_{i=0}^{\left\llcorner T_{\gamma_{n}} / T\right\lrcorner-1} \operatorname{det}\left(\left.D \phi^{i T}(x)\right|_{\mathcal{N}_{L}^{s} \oplus<X_{n}\left(x_{n}\right)>}\right) \leq e^{-\nu T_{\gamma_{n}}},
$$

where $T_{\gamma_{n}}$ is the period of $\gamma_{n}$.

Then for any $\gamma_{n}$, taking $T=1$, Pliss Lemma (remark 12) gives some point $p_{n} \in \gamma_{n}$ satisfying 


$$
\frac{1}{k} \sum_{i=0}^{k / 1} \log \left(\operatorname{det}\left(\left.D \phi_{n}^{1}\right|_{D \phi^{i}\left(\mathcal{N}_{L}^{s} \oplus<X_{n}\left(p_{n}\right)>\right)}\right)\right) \leq-\nu .
$$

Assume $p_{n}$ tends to $y \in \Gamma \cup \sigma$. One can assume $\mathcal{N}_{L}^{s} \oplus<X_{n}(x n)>\rightarrow E(y)$, and since $y$ is accumulated by Pliss points, again we have that:

$$
\frac{1}{k} \sum_{i=0}^{k} \log \left(\operatorname{det}\left(\left.D \phi^{1}\right|_{D \phi^{i}(E(y))}\right)\right) \leq-\nu,
$$

Now the Pliss Lemma again, allows us to find $n_{j} \rightarrow \infty$ such that

$$
\frac{1}{k} \sum_{i=0}^{k} \log \left(\operatorname{det}\left(\left.D \phi^{1}\right|_{D \phi^{i+n_{j}}(E(y))}\right)\right) \leq-\nu .
$$

Since $\phi^{i+n_{j}}(y)$ tends to $\sigma$ as $n_{j} \rightarrow \infty$, we derive a subspace $E \subset T_{\sigma} M$ with $\operatorname{dim}(E)=n+1$ such that

$$
\frac{1}{k} \sum_{i=0}^{k / 1} \log \left(\operatorname{det}\left(\left.D \phi^{1}\right|_{D \phi^{i}(E)}\right)\right) \leq-\nu .
$$

This shows that $E$ contracts volume, and the proof is analogous for $F$

The following corollary is a consequence of Lemma 25.

Corollary 26. There is Let $\mathcal{V}$ be the set of $\mathcal{C}^{1}$ vector fields $X$ with a robustly chain transitive class $C$ and such that:

- Every singularity in $C$ is hyperbolic and

$$
T_{\sigma} M=E^{s s} \oplus E^{c} \oplus E^{u u},
$$

notes the stable escaping, the unstable escaping and the center spaces.

- there is with a finest dominated splitting over $\widetilde{\Lambda}(X, U)$

$$
\mathcal{N}_{L}=\mathcal{N}^{s} \oplus \mathcal{N}^{1} \oplus \cdots \oplus \mathcal{N}^{k} \oplus \mathcal{N}^{u} .
$$

where $U$, an isolating neighborhood of $C$, and $n=\operatorname{dim}\left(\mathcal{N}^{s}\right)>\operatorname{dim}\left(E^{s s}\right)$

There is an open and dense subset $\mathcal{U} \subset \mathcal{V}$ such that for every $X \in \mathcal{U}$ every singularity in $C$ is such that there is a $n+1$ dimensional space $E \subset T_{\sigma} M$ that contracts volume. Moreover $E^{s s} \oplus E^{c} \subset E$

Proof. Let a vector field $X$ be a vector field such that

- Every singularity in $C$ is hyperbolic and

$$
T_{\sigma} M=E^{s s} \oplus E^{c} \oplus E^{u u},
$$

notes the stable escaping, the unstable escaping and the center spaces. 
- there is with a finest dominated splitting over $\widetilde{\Lambda}(X, U)$

$$
\mathcal{N}_{L}=\mathcal{N}^{s} \oplus \mathcal{N}^{1} \oplus \cdots \oplus \mathcal{N}^{k} \oplus \mathcal{N}^{u} .
$$

where $U$, an isolating neighborhood of $C$, and $n=\operatorname{dim}\left(\mathcal{N}^{s}\right)>\operatorname{dim}\left(E^{s s}\right)$

- all periodic orbits in $C$ are hyperbolic

Note that this is a dense subset of the vector fields $X$ with a robustly chain transitive class $C$.

By the connecting Lemma 9 we can find a vector field $Y$ that is $\epsilon-C^{1}$ close to $Y^{\prime}$ and is equal to $X$ in a neighborhood of $\sigma$, such that there is $\Gamma=\operatorname{Orb}(x)$ a homoclinic orbit associated to $\sigma$. Now Theorem 10 allow us to find a sequence of vector fields

- there exists a sequence of star vector fields $Y_{n}$ converging to $Y$ in the $C^{1}$ topology

- there exist a sequence of periodic orbit $\gamma_{n}$ of $Y_{n}$ such that $\gamma_{n}$ converges to $\Gamma$ in the Hausdorff topology.

- there is a sequence of points $q_{n} \in \gamma_{n}$ such that $Y_{n}\left(q_{n}\right) \rightarrow u$ is in $E^{u 1} \oplus$ $\cdots \oplus E^{u l}$.

- we call $L_{u}=<u>$ and we have that $\operatorname{dim}\left(\mathcal{N}_{L_{u}}^{u}\right)=h$

- There is a sequence of points $p_{n} \in \gamma_{n}$ such that $\left\langle Y_{n}\left(p_{n}\right)\right\rangle \rightarrow v$ is in $E^{s 1} \oplus \cdots \oplus E^{s n}$

- we call $L_{v}=\left\langle v>\right.$ and we have that $\operatorname{dim}\left(\mathcal{N}_{L_{v}}^{s}\right)=n$.

Since $Y$ is now in the conditions of lemma25then there is an invariant space $E$ of dimension $n+1$ that contracts volume. Since from corollary $23 \operatorname{dim}\left(E^{s s} \oplus E^{c}\right) \leq$ $n+1$, then $E^{s s} \oplus E^{c} \subset E$.

Since in a neighborhood of a singularity $X$ and $Y$ are equal, and since the volume contraction of a subspace of $T_{\sigma} M$ is an open property, we get our result.

As a direct consequence we have:

Corollary 27. Let $\sigma$ be a singularity of $C$, a robustly chain transitive class with all singularities hyperbolic, with a finest dominated splitting over $\widetilde{\Lambda}$

$$
\mathcal{N}_{L}=\mathcal{N}^{s} \oplus \mathcal{N}^{1} \oplus \cdots \oplus \mathcal{N}^{k} \oplus \mathcal{N}^{u} .
$$

Suppose that $n=\operatorname{dim}\left(\mathcal{N}^{s}\right)>\operatorname{dim}\left(E^{s s}\right)$ then $E^{c s}=E^{s s} \oplus E_{\sigma}^{c}$ contracts volume.

The following corollary summarizes the situation

Corollary 28. Let $\sigma$ be a singularity in our chain recurrent class. We define $E^{c s}=E^{s s} \oplus E_{\sigma}^{c}$. We have 2 possibilities,

- either $\pi_{L}\left(E_{\sigma}^{c}\right) \cap \mathcal{N}_{L}^{s}=\emptyset$, and then there is a space $\mathcal{N}^{\text {ss }}$ over the singularity contracts uniformly or

- $E^{c s} \subset \mathcal{N}^{s s} \oplus L \subset E$ and $E$ contracts volume for $L \in E_{\sigma}^{c}$. 


\section{Proof of the main theorem}

We aim now to prove Theorem 5 The proof is very similar to the proof in Ma2. In fact is an adaptation to flows of the proof of theorem 4 in BDP. The idea is to argue by contradiction and show that if there is no uniform volume expansion in the extremal bundle, then there is a closed orbit orbit of a sufficiently close vector field that contracts volume in the extremal bundle. This could be a periodic orbit or a singularity, but sections 3.2 and 5.1 show us that this is not possible.

The following proposition is equivalent to lemma 6.5 form $[\mathrm{BDP}$, and the proof is analogous.

Lemma 29. Let $X \in \mathcal{X}^{1} M$ be a vector field, $C$ a maximal invariant in a filtrating neighborhood $U \subset M$ Suppose there is a dominated splitting $E \oplus \prec F$ over $\widetilde{\Lambda}(X, U)$ for the reparametrized linear Poincaré flow, $h_{E c}^{T} \cdot \psi^{T}(L)$. Then if the Jacovian of $h_{E c}^{T} \cdot \psi^{T}(L)$ restricted to $E$ is not bounded from above by one, then for every $T$ there is a $h_{E c}^{T} \cdot \psi^{T}(L)$ invariant measure $\nu$ such that

$$
\int \log \left|J\left(h_{E c}^{T} \cdot \psi^{T}(L), E\right)\right| d \nu \geq 0
$$

Now we want to show that if $h_{E c}^{T} \cdot \psi^{T}(L)$ does not contract volume on the most dominated bundle of the finest dominated splitting in $\widetilde{\Lambda}(X, U)$, then, the measure $\nu$ from the previous lemma is not supported on the directions that are over the singularities.

The following lemma is a consequence of corollary 26.

Lemma 30. Let $\mathcal{V} \subset \mathcal{X}^{1} M$ be the set of vector fields $X$ such that

- it has a robustly chain transitive class $C$ that is maximal invariant in a filtrating neighborhood $U \subset M$ with a singularity $\sigma$

- there is a finest dominated splitting

$$
\mathcal{N}_{L}=\mathcal{N}_{L}^{s} \oplus \prec \cdots \oplus \prec \mathcal{N}_{L}^{u}
$$

over $\widetilde{\Lambda}(X, U)$ for the reparametrized linear Poincaré flow, $h_{E c}^{T} \cdot \psi^{T}(L)$

Them there is an open and dense subset $\mathcal{U} \subset \mathcal{V}$ such that for any $X \in \mathcal{U}$ any $h_{E c}^{T} \cdot \psi^{T}(L)_{T}$ invariant measure $\nu$ supported in $\mathbb{P}_{\sigma}^{c} \cap \widetilde{\Lambda}(X, U)$ is such that

$$
\int \log \left|J\left(h_{E c}^{T} \cdot \psi^{T}(L)_{T}, \mathcal{N}_{L}^{s}\right)\right| d \nu<0
$$

Proof. Let us start by supposing that $\sigma \in S_{E}$ and $\operatorname{dim}\left(\mathcal{N}^{s}\right) \leq E^{s s}$. In this case, we can include $\mathcal{N}^{s} \subset T \sigma M$ as a subspace of $E^{s s}$. Then $\mathcal{N}^{s}$ contracts uniformly for the tangent space and for the extended linear Poincaré flow. Note that in this case the reparametrized linear Poincaré flow and the extended linear Poincaré flow are equal in restriction to $\operatorname{dim}\left(\mathcal{N}^{s}\right)$ at the directions over $\sigma$. 
Suppose that $\sigma \in S_{E c}$ and $n_{s}=\operatorname{dim}\left(\mathcal{N}^{s}\right) \geq E^{s s}$, then given $L \in \mathbb{P}_{\sigma}^{c} \cap$ $\widetilde{\Lambda}(X, U)$ there exist a subspace $E=\mathcal{N}^{s} \oplus L$ of $T_{\sigma} M$ that contracts volume, The reparametrized linear Poincaré flow at the directions over $\sigma$ is $h_{E c}^{T} \cdot \psi^{T}(L)$ where

$$
h_{E c}^{T}=\left(\frac{\left\|d \phi^{t}(u)\right\|}{\|u\|}\right)^{\frac{1}{n_{s}}}
$$

for a non vanishing vector $u$ in the direction of $L$. Then

$$
\left|J\left(\psi_{T}, \mathcal{N}_{L}^{s}\right)\right|\left(\frac{\left\|d \phi^{t}(u)\right\|}{\|u\|}\right)=\left|J\left(d \phi_{T}, E\right)\right| .
$$

In any case lemma 29 allows us to conclude.

The flowing lemma is the only missing piece for Theorem 5 for $\widetilde{\Lambda}(X, U)$. Untill now we have from lemma 29 that if there is no volume contraction of $\mathcal{N}_{L}^{S}$, then there is a measure showing this lack of contraction. From lemma 30 we also know that this measure can not be supported over a singularity. Finally the next lemma uses the ergodic closing lemma to prove that if a measure was showing the lack of contraction, then it would be supported on a singularity contradicting the previous lemma. So by contradiction the following lemma implies the volume contraction of the least dominated bundle. For the volume expansion the proof is analogus. Later we extend this structure over $\widetilde{\Lambda}(X, U)$ to $B(C)$ and conclude with the proof of Theorem 5 .

Lemma 31. Let $X \in \mathcal{X}^{1} M$ be a vector field, $L_{a}$ a maximal invariant in a filtrating neighborhood $U \subset M$ and the set $\widetilde{\Lambda}$. Suppose there is a finest dominated splitting

$$
\mathcal{N}_{L}=\mathcal{N}_{L}^{s} \oplus_{\prec} \cdots \oplus \prec \mathcal{N}_{L}^{u}
$$

over $\widetilde{\Lambda}(X, U)$ for the reparametrized linear Poincaré flow, $h_{E c}^{T} \cdot \psi^{T}(L)$. If there is a $h_{E c}^{T} \cdot \psi^{T}(L)$ invariant measure $\nu$ such that

$$
\int \log \left|J\left(h_{E c}^{T} \cdot \psi^{T}(L), \mathcal{N}_{L}^{s}\right)\right| d \nu \geq 0,
$$

then the measure must be supported on $\mathbb{P}_{\sigma}^{c} \cap \widetilde{\Lambda}(X, U)$.

Proof.

Claim. Let $\nu_{n}$ be a measure supported on a periodic orbits $\gamma_{n}$ with period $\pi \gamma_{n}$ bigger than $T$, then $\int \log h_{E c}^{n_{s} \cdot T} d \nu_{n}(x)=0$.

Proof. By definition of $h_{E c}^{T}$

$$
\log h_{E c}^{n_{s} \cdot T} d \nu_{n}(x)=\log \Pi_{\sigma_{i} \in S_{E c}}\left\|h_{\sigma_{i}}^{n_{s} \frac{1}{n_{s}} T}\right\| d \nu_{n}(x),
$$

so it suffices to prove the claim for a given $h_{\sigma_{i}}^{T}$. For every $x$ in $\gamma$, since $h_{\sigma_{i}}^{T}$ is a multiplicative cocycle we have that: 


$$
\Pi_{i=0}^{(m \pi(\gamma) / T)-1} \quad h_{\sigma_{i}}^{T}\left(\phi_{i T}^{Y}(x)\right)=h_{\sigma_{i}}^{(m \pi(\gamma) / T)-1}(x)
$$

The norm of the vector field restricted to $\gamma$ is bounded, and therefore $h_{\sigma_{i}}^{(m \pi(\gamma) / T)^{-1}}(x)$ is bounded for $m \in \mathbb{N}$ going to infinity. Then this is also true for $h_{E c}^{T}$. Since $\nu_{n}$ is an ergodic measure, we have that

$$
\begin{aligned}
\int \log h_{\sigma_{i}}^{T}(x) d \nu_{n}(x) & =\lim _{m \rightarrow \infty} \frac{1}{m} \sum_{i=0}^{(m \pi(\gamma) / T)-1} \log \left(h_{\sigma_{i}}^{T}\left(\phi_{i T}^{Y}(x)\right)\right) \\
& =\lim _{m \rightarrow \infty} \frac{1}{m} \log \left(\Pi_{i=0}^{(m \pi(\gamma) / T)-1} h_{\sigma_{i}}^{T}\left(\phi_{i T}^{Y}(x)\right)\right) \\
& =\lim _{m \rightarrow \infty} \frac{1}{m} \log \left(h_{\sigma_{i}}^{(m \pi(\gamma) / T)-1}(x)\right) \\
& =0
\end{aligned}
$$

Suppose that $\mu$ weights 0 on

$$
\bigcup_{\sigma_{i} \in \operatorname{Sing}(X)} \mathbb{P}_{\sigma_{i}}^{c} \cap \widetilde{\Lambda}(X, U)
$$

then $\mu$ projects on $M$ on an ergodic measure $\nu$ supported on the class $C$ and such that ut weights 0 in the singularities, for which

$$
\int \log \left|J\left(h_{E} \cdot \psi_{\mathcal{N}}^{T}, \mathcal{N}^{s}\right)\right| d \nu(x) \geq 0 .
$$

Recall that $\psi^{T}$ is the linear Poincaré flow, and $h_{E c}^{T}$ can be defined as a function of $x \in M$ instead of as a function of $L \in \mathbb{P} M$ outside of an arbitrarily small neighborhood of the singularities.

However, the ergodic closing lemma implies that $\nu$ is the weak*-limit of measures $\nu_{n}$ supported on closed orbits $\gamma_{n}$ which converge for the Hausdorff distance to the support of $\nu$. Therefore, for $n$ large enough, the $\gamma_{n}$ are contained in $C$ and from remark 26 and our previous claim we know that

$$
\begin{aligned}
\left.\int \log \left|J\left(h_{E c}^{T} \cdot \psi_{\mathcal{N}}^{T}, \mathcal{N}^{s}\right)\right| d \nu_{n}(x)\right) & =\int \log \left|J\left(\psi_{\mathcal{N}}^{T}, \mathcal{N}^{s}\right)\right| d \nu_{n}(x) \\
\int \log \left|J\left(\psi_{\mathcal{N}}^{T}, \mathcal{N}^{s}\right)\right| d \nu_{n}(x) & \leq-\eta
\end{aligned}
$$

Then

$$
\left.\int \log \left|J\left(h_{E c}^{T} \cdot \psi_{\mathcal{N}}^{T}, \mathcal{N}^{s}\right)\right| d \nu_{n}(x)\right) \leq-\eta
$$

The map $\log \left|J\left(h_{E c} \cdot \psi_{\mathcal{N}}^{T}, \mathcal{N}^{s}\right)\right|$ is not continuous. Nevertheless, it is uniformly bounded and the unique discontinuity points are the singularities of $X$. These 
singularities have (by assumption) weight 0 for $\nu$ and thus admit neighborhoods with arbitrarily small weight. Out of such a neighborhood the map is continuous. One deduces that

$$
\int \log \left|J\left(h_{E c} \cdot \psi_{\mathcal{N}}^{T}, \mathcal{N}^{s}\right)\right| d \nu(x)=\lim \int \log \left|J\left(h_{E c} \cdot \psi_{\mathcal{N}}^{T}, \mathcal{N}^{s}\right)\right| d \nu_{n}(x)
$$

and therefore is strictly negative, contradicting the assumption.

Note that all of this is also valid for the reverse time of the flow and for $\mathcal{N}^{u u}$. Lemmas 30 and 31 and their versions for the reverse time implies Theorem 5 over $\widetilde{\Lambda}(X, U)$. We re state it as the following corollary

Corollary 32. Let $\mathcal{V} \subset \mathcal{X}^{1} M$ be the set of vector fields $X$ such that it has a robustly chain transitive class $C$ that is maximal invariant in a filtrating neighborhood $U \subset M$ with a singularity $\sigma$

Them there is an open and dense subset $\mathcal{U} \subset \mathcal{V}$ such that for any $X \in \mathcal{U}$ is singular volume partial hyperbolic over $\widetilde{\Lambda}(X, U)$.

Theorem 4 from $\mathrm{BdL}$ gives immediately the following, which is equivalent to Theorem 5 ,

Corollary 33. Let $\mathcal{V} \subset \mathcal{X}^{1} M$ be the set of vector fields $X$ such that it has a robustly chain transitive class $C$ that is maximal invariant in a filtrating neighborhood $U \subset M$ with a singularity $\sigma$

Them there is an open and dense subset $\mathcal{U} \subset \mathcal{V}$ such that for any $X \in \mathcal{U}$ is singular volume partial hyperbolic over $B(C)$.

Corollary 34. Let $X$ be a vector field with a robustly chain transitive attractor $C$ in the attracting set $U$ and all singularities in $C$ are hyperbolic. Then $C$ is singular volume partial hyperbolic. Moreover $C$ has a dominated splitting over $\widetilde{\Lambda}(X, U)$ of the form $\mathcal{N}_{L}=E \oplus F$ where $E$ is contracting and $F$ is volume expanding for the reparametrized linear poincaré flow.

Proof. Since $C$ is an attractor then $C$ has a dominated splitting over $\widetilde{\Lambda}(X, U)$ of the form $\mathcal{N}_{L}=E \oplus F$ where $E$ is contracting. Given any singularity $\sigma$ in $C$ the center space $E^{c}$ projects to $F$ for all directions of $\widetilde{\Lambda}(X, U)$ over $\sigma$. Therefore by Lemma 23 the finest dominated splitting of $C$ has $F$ as the dominating extremal bundle. Therefore by Corollary $33 F$ has to be volume expanding for the reparametrized linear Poincaré flow.

Remark 29. Note that since the example in [BLY] is in de conditions of corolary 33 then it is singular volume partial hyperbolic. 


\section{References}

[AN] John M. Alongi, Gail Susan Nelson Recurrence and Topology, Volume 85 of Graduate studies in mathematics, ISBN 0821884050, 9780821884058

[BB] J. Bochi, C. Bonatti; Perturbation of the Lyapunov spectra of periodic orbits. Proc London Math Soc 2012; 105 (1): 1-48

[BC] C. Bonatti and S. Crovisier, Recurrence et genericité, Invent. Math., 158 (2004), 33-104.

[BDP] C. Bonatti, L. J. Díaz and E. R. Pujals, A C1-generic dichotomy for diffeomorphisms: Weak forms of hyperbolicity or infinitely many sinks or sources, Annals of Math. (2), 158 (2003), 355-418.

[BDV] C. Bonatti, L. J. Díaz, and M. Viana Dynamics beyond uniform hyperbolicity. A global geometric and probabilistic perspective. Encyclopaedia of Mathematical Sciences, 102. Mathematical Physics, III. Springer-Verlag, Berlin, (2005). xviii+384 pp.

[BGY] C. Bonatti, S. Gan and D. Yang, Dominated chain recurrent classes with singularities, arXiv:1106.3905.

[BGV] C. Bonatti, N.Gourmelonn and T. Vivier, Perturbations of the derivative along periodic orbits. Ergodic Theory and Dynamical Systems, 26(5), 13071337.(2006)

[BdL] C. Bonatti, A. da Luz Star flows and multisingular hyperbolicity preprinr arXiv:1705.05799

[BLY] C. Bonatti, M. Li, and D. Yang, A robustly chain transitive attractor with singularities of different indices. J. Inst. Math. Jussieu 12 (2013), no. 3, 449-501.

[BaMo] S Bautista, CA Morales On the intersection of sectional-hyperbolic sets arXiv preprint arXiv:1410.0657

[BPV] C.Bonatti, A. Pumariño, and M. Viana, Lorenz attractors with arbitrary expanding dimension. C. R. Acad. Sci. Paris Sér. I Math. 325 (1997), no. $8,883-888$.

[BV] C.Bonatti, Christian; M. Viana, SRB measures for partially hyperbolic systems whose central direction is mostly contracting. Israel J. Math. 115 (2000), 157-193.

[Co] C. Conley, Isolated Invariant Sets and the Morse Index, CBMS Regional Conference Series in Mathematics, vol. 38, American Mathematical Society, Rhode Island, 1978. 
[C] S. Crovisier, Periodic orbits and chain-transitive sets of C1diffeomorphisms, Publ. Math. Inst. Hautes études Sci., 104 (2006), 87-141.

[D] C.I. Doering, Persistently transitive vector fields on three-dimensional manifolds. Dynamical systems and bifurcation theory (Rio de Janeiro, 1985), 59-89, Pitman Res. Notes Math. Ser., 160, Longman Sci. Tech., Harlow, 1987.

$[\mathrm{dM}]$ W.de Melo, Structural stability of diffeomorphisms on two-manifolds. Invent. Math. 21 (1973), 233-246.

[DPU] L. J. Díaz, E. Pujals, and R. Ures, Partial hyperbolicity and robust transitivity. Acta Math. 183 (1999), no. 1, 1-43.

[GY] S. Gan and D. Yang, Morse-Smale systems and horseshoes for threedimensional singular fows, arXiv:1302.0946.

[G] J. Guckenheimer, A strange, strange attractor, in The Hopf Bifurcation Theorems and its Applications, Applied Mathematical Series, 19, SpringerVerlag, 1976, 368-381.

$[\mathrm{H}]$ S. Hayashi, Diffeomorphisms in $F^{1}(M)$ satisfy Axiom A, Ergod. Th. Dynam. Sys., 12 (1992), 233-253.

[H2] S. Hayashi, Connecting invariant manifolds and the solution of the $C^{1}$ stability conjecture and $\omega$-stability conjecture for flows, Ann. of Math., 145 (1997), 81-137.

[K] A. Katok, Lyapunov exponents, entropy and periodic orbits for diffeomorphisms, Inst. Hautes Etudes Sci. Publ. Math., 51 (1980), 137-173.

[GLW] M. Li, S,Gan, L.Wen, Robustly Transitive singular sets via approach of an extended linear Poincaré flow. Discrete and Continuous Dynamical System. Volume 13, Number 2, July 2005

[GSW] Y. Shi, S,Gan, L.Wen, On the singular hyperbolicity of star flows. Journal Of Modern Dynamics. October 2013.

[GWZ] S. Gan, L. Wen and Zhu,Indices of singularities of robustly transitive sets, Discrete Contin. Dyn. Syst., 21 (2008), 945-957.

[GW] J. Guchenheimer and R. Williams, Structural stability of Lorenz attractors, Inst. Hautes Etudes Sci. Publ. Math., 50 (1979), 59-72.

[L] S. Liao, On $(\eta, d)$-contractible orbits of vector fields, Systems Sci. Math. Sci., 2 (1989), 193-227.

[Lo] E. N. Lorenz, Deterministic nonperiodic flow, J. Atmosph. Sci., 20 (1963), 130-141. 
[Ma] R. Mañé, An ergodic closing lemma, Ann. Math. (2), 116 (1982), 503-540

[Ma1] R. Mañé, Contributions to the stability conjecture. Topology 17 (1978), no. $4,383-396$.

[Ma2] R. Maǹé, A proof of the $C^{1}$ stability Conjecture, Publ. Math. IHES, 66 (1988), 161-210

[MM] R. Metzger and C. Morales, On sectional-hyperbolic systems, Ergodic Theory and Dynamical Systems, 28 (2008), 1587-1597.

[MPP] C. Morales, M. Pacifico and E. Pujals, Robust transitive singular sets for 3-?ows are partially hyperbolic attractors or repellers, Ann. Math. (2), 160 (2004), 375-432.

[PaSm] J. Palis and S. Smale, Structural stability theorems, in 1970 Global Analysis (Proc. Sympos. Pure Math., Vol. XIV, Berkeley, Calif., 1968), Amer. Math. Soc., Providence, R.I, 1970, 223-231.

[Pl] V. Pliss, A hypothesis due to Smale, Diff. Eq., 8 (1972), 203-214.

[PuSh] C. Pugh and M. Shub, w-stability for flows, Invent. Math., 11 (1970), $150-158$.

[PuSh2] C. Pugh and M. Shub, Ergodic elements of ergodic actions, Compositio Math., 23 (1971), 115-122.

[R1] J. W. Robbin, A structural stability theorem. Ann. of Math. (2) 94 (1971) 447-493.

[R2] C. Robinson, Structural stability of $C^{1}$ diffeomorphisms. J. Differential Equations 22 (1976), no. 1, 28-73.

[Sh] M. Shub, Topologically transitive diffeomorphisms on $T^{4}$ In Dynamical Systems, volume 206 of Lecture Notes in Math., lecture (16) page 28-29 and lecture (22) page 39, Springer Verlag, 1971.

[Sm] S. Smale, The $\omega$-stability theorem, in 1970 Global Analysis (Proc. Sympos. Pure Math., Vol. XIV, Berkeley, Calif., 1968), Amer. Math. Soc., Providence, R.I., 1970, 289-297.

[V] T.Vivier,Flots robustement transitifs sur les variétés compactes. Comptes Rendus Acad. Sci. Paris, 337:791-796, 2003.

[W] L. Wen, On the C1 stability conjecture for flows, J. Differential Equations, 129 (1996), 334- 357.

[WX] L. Wen and Z. Xia, C1 connecting lemmas, Trans. Am. Math. Soc., 352 (2000), 5213-5230.

[YZ] D. Yang and Y. Zhang, On the finiteness of uniform sinks, J. Diff. Eq., 257 (2014), 2102-2114. [32] S. 